\title{
Desnutrición infantil y tenencia de la tierra en Cuscatlán seis años después de los acuerdos de paz: un estudio epidemiológico
}

\author{
Paula E. Brentlinger \\ Miguel A. Hernán \\ Sonia Hernández-Díaz
}

\section{Introducción}

La desnutrición infantil y la pobreza son parientes. Cuando la familia cuenta con los recursos necesarios para cultivar o adquirir alimentos, los padres poseen una educación que les permite seleccionar apropiadamente la dieta de sus hijos, hay agua suficiente para mantener la higiene que

\section{Resumen}

El programa de reconstrucción de las zonas devastadas por la guerra civil salvadoreña debería, a estas alturas, haber reducido los niveles de pobreza y de desnutrición infantil. Sin embargo, seis años después de la firma de los acuerdos de paz, ha sido evidente que la ejecución del plan nacional de reconstrucción no ha sido uniforme. La prevalencia elevada de desnutrición infantil está relacionada con las demoras en la implementación de los acuerdos socioeconómicos de Chapultepec.

1. "Prevalencia" es el porcentaje de personas de la población que padecen una enfermedad en un momento determinado. Por ejemplo, si en un grupo de 100 personas 10 tienen fiebre tifoidea hoy, la prevalencia de tifoidea en este grupo ahora es del 10 por ciento, independientemente de cuándo enfermaron o de si otras personas del grupo tuvieron tifoidea antes pero ya están recuperadas. 
impacto de los programas, cuyo objetivos son la reducción de la pobreza ${ }^{2}$.

El programa de reconstrucción de las zonas devastadas por la guerra civil salvadoreña debería, en principio, haber reducido los niveles de pobreza y desnutrición infantil en las poblaciones beneficiadas. La transferencia de tierra y la restauración de servicios básicos incluidos en el Acuerdo de Chapultepec, deberían haber aumentado la capacidad de los campesinos beneficiarios para garantizar una vida sana a sus niños ${ }^{3}$.

Seis años después de la firma de los acuerdos de paz, era evidente que la implementación del plan nacional de reconstrucción no había sido uniforme. Algunos beneficiarios del Plan de Transferencia de Tierras (PTT) lograron comprar y cultivar sus nuevas tierras en los primeros años de la paz; otros aún estaban embarcados en las negociaciones de compra y venta. Algunas comunidades habían recibido viviendas, o escuelas, o sistemas de agua; otras se quedaron casi sin nada. Tan diferentes fueron los patrones de ejecución del plan de reconstrucción, que nuestro grupo de investigadores decidió utilizar este "experimento natural" para evaluar la relación entre los distintos patrones y el estado de salud nutricional de los niños beneficiarios del programa. Nuestras dos hipótesis principales eran: (1) La prevalencia de desnutrición infantil (baja talla-para-edad) se mantiene elevada a pesar de los programas de reconstrucción. (). La alta prevalencia de desnutrición infantil (baja tallapara-edad) está relacionada con las demoras en la implementación de los acuerdos socioeconómicos de Chapultepec.

\section{Indicadores de desnutrición}

Muchos programas de salud y asistencia social en El Salvador vigilan el crecimiento de niños menores de 5 años, pero son pocos los programas que utilizan la medida talla-para-edad. Otros dos indicadores, peso-para-edad y peso-para-talla, son más conocidos y se utilizan con más frecuencia. ¿Por qué, entonces, se usa el indicador menos conocido?

El retardo del crecimiento lineal, o baja tallapara-edad, es la forma más común de desnutrición infantil en El Salvador y en el resto de Centroamérica. Según los resultados preliminares del estudio FESAL-984, la prevalencia actual de baja talla-para-edad en El Salvador es de 23.1 por ciento, frente a $\mathbf{1 1 . 1}$ por ciento de bajo peso-para-edad y 1.2 por ciento de bajo peso-para-talla. Como puede verse, la prevalencia de baja talla-para-edad es casi 20 veces más alta que la prevalencia de bajo peso-para-talla. Un patrón similar se encontró en 1993, fecha en que se llevó a cabo la penúltima encuesta nacional de salud familiar, cuando la prevalencia de baja talla-para-edad fue de 22.0 por ciento, la de bajo peso-para-talla de 2.9 por ciento, y la de bajo peso-para-edad de 10.5 por ciento. Los datos para $1988^{5}$ se pueden ver en la Figura 1.

Figura 1

\section{Desnutrición en El Salvador 1988-1998}

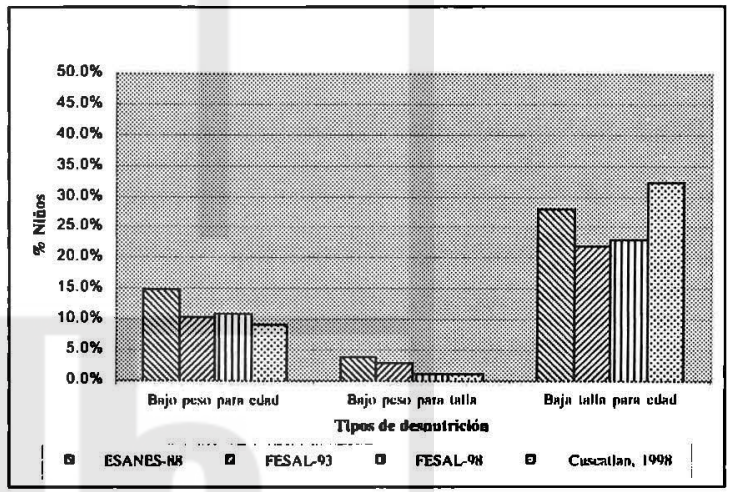

La emaciación, o bajo peso-para-talla, indica una deficiencia aguda de alimentación (inmediatamente después de una inundación o un desplazamiento masivo de refugiados, por ejemplo), o una pérdida aguda de líquidos corporales en el niño (a causa de diarrea aguda o fiebre alta, por ejemplo).

2. World Health Organization Expert Committee on Physical Status. Physical Status: The Use and Interpretation of Anthropometry, Geneva, Switzerland: World Health Organization, 1995. WHO Technical Report Series 854.

3. Acuerdo de Paz de Chapultepec, Capítulo V, Tema Ecónomico y Social.

4. L. Grummer-Strawn, Resultados preliminares sobre nutrición de la encuesta nacional de salud familiar, 1998. Presentación realizada con motivo de la Conferencia Nacional de Nutrición de El Salvador, San Salvador, del 14-15 de enero de 1999.

5. L. Grummer-Strawn, J. Cáceres y B. Herrera, "Trends in the nutritional status of Salvadorean children: the postwar experience", Bull World Health Organ, 1996, 74, pp. 369-374. 
Por lo tanto, el índice peso-para-talla es un indicador muy útil a nivel hospitalario o para calcular el estado agudo de una población recientemente afectada por un desastre.

Sin embargo, cuando la falta de alimentación y/o los efectos de enfermedades infecciosas son crónicos (a largo plazo), el cuerpo del niño afectado se adapta a las nuevas condiciones y su ritmo de crecimiento disminuye. Después de un período de meses (o años) de desnutrición crónica, el peso del niño puede adecuarse a su talla, pero la talla no es la esperada para su edad. El niño no parece delgado, sino simplemente más bajo de lo que le correspondería a su edad.

El bajo peso-para-edad es una medida que combina los componentes de desnutrición aguda (emaciación) y crónica (baja talla) en un solo número y es, por tanto, de utilidad más limitada, pues no permite evaluar la contribución relativa de cada uno de ellos al estado nutricional del niño.

Podría pensarse que la raza es un factor muy importante en lo que a la altura del niño se refiere y que, por tanto, algunas razas humanas serán siempre más altas que otras. Sin embargo, se ha demostrado que en los países en vías de desarrollo, los principales determinantes del crecimiento del niño son ambientales, no genéticos. De hecho, antes de los 5 años de

El retardo del crecimiento lineal, o baja talla-para-edad, es la forma más común de desnutrición infantil en El Salvador y en el resto de Centroamérica. edad, el ritmo de crecimiento - determinado por la talla - en niños bien nutridos es igual o casi igual a nivel internacional ${ }^{6}$. Por ejemplo, cuando familias de refugiados emigran de paises pobres hacia otros con más recursos, la primera genera- ción de niños nacidos en mejores condiciones es una generación más alta que sus padres. Asimismo, cuando un país pobre comienza a desarrollarse, cada nueva generación es más alta que la anterior por haber tenido una mejor alimentación y menos infecciones. Un caso muy conocido es el de los japoneses, tradicionalmente considerados como una raza de baja estatura y que actualmente tienen una estatura media cercana a la occidental.

Se sabe muy bien que un niño con bajo peso y débil tiene un mayor riesgo de morir por desnutrición o enfermedad. El niño de baja talla, aunque no esté delgado, también corre serios riesgos. Investigaciones científicas en Centroamérica ( $y$ otros lugares) muestran con claridad que es más probable que el niño de baja talla tenga un bajo rendimiento escolar durante la niñez, así como un bajo rendimiento laboral en la edad adulta ${ }^{78910}$. La reducción de la capacidad productiva del adulto atribuible al retardo del crecimiento infantil es tal, que El Salvador podría perder 408 millones de colones en los próxi-

6. World Health Organization Expert Committee on Physical Status, op. cit.

7. L.H. Allen, "Malnutrition and human function: a comparison of conclusions from the INCAP and Nutrition CRSP studies", J Nutr, 125 (suppl. 4), 1995, pp. 1119S-1126S.

8. E. Pollitt, K. Gorman, P. Engle et al, "Nutrition in early life and the fulfillment of intellectual potential", $J$ Nutr, 125 (suppl. 4), 1995, pp. 1111S-1118S.

9. J. Haas, S. Murdoch, J. Rivera et al, "Early nutrition and later physical work capacity", Nutr Rev, 54 (suppl. 2 pt 2), 1996, pp. S41-S48.

10. R. Martorell, "The role of nutrition in economic de development", Nutr Rev, 54 (suppl 4 pt 2), 1996, pp. S66S71. 
mos 5 años (período 1999-2003) si no soluciona el problema ${ }^{11}$. Afortunadamente, otras investigaciones demuestran que la identificación de niños de alto riesgo y la intervención con alimentos y atención médica adecuada, podría ayudar ${ }^{12}$ a reducir los efectos.

La prevalencia de baja talla como indicador del impacto de los programas de desarrollo, se ha recomendado porque se espera un descenso en la prevalencia de baja talla al contar con agua potable, saneamiento ambiental, buena alimentación, prevención y tratamiento de enfermedades infecciosas ${ }^{1314}$ is 16171819 . Indicadores sobre el proceso, como la cantidad de dinero invertido en la capacitación de personal médico o en la compra de cemento y lámina, o el número de escrituras emitidas, pueden ser útiles también en la vigilancia de programas, pero no nos dicen nada sobre si realmente la implementación de un programa ha sido eficaz para los beneficiarios. Un indicador de impacto - por ejemplo, algo que mide el estado actual de salud (mortalidad o morbilidad) del niño- puede informamos si el proceso realmente logra llegar a su meta final: mejorar la vida del beneficiario.

A continuación presentamos los métodos de este estudio y los resultados más relevantes ${ }^{20}$.

\section{Métodos}

El estudio fue diseñado con la colaboración de parteras, promotores de salud, enfermeras y médi- cos de un municipio del departamento de Cuscatlán. Este municipio, despoblado y casi destruido durante la guerra civil, comenzó a repoblarse en 1986. Identificamos 37 comunidades rurales repobladas o establecidas antes de 1994 , de las cuales escogimos una muestra representativa de 27 . La población estudiada estaba compuesta por niños menores de 5 años que vivían con sus familias en las 27 comunidades, en enero de 1998.

Un equipo de 4 investigadores visitaron las comunidades en enero de 1998, acompañados por parteras y promotores de salud locales, que identificaron las casas donde vivían niños menores de 5 años. En las comunidades más pequeñas, el equipo visitó todas las casas en donde habían niños menores de 5 años. En las comunidades más grandes se seleccionó una muestra al azar (superior al 50 por ciento del total de niños en todas, menos en una comunidad). El 69 por ciento de todas las casas que se eligieron se visitaron. Ninguna familia rechazó la entrevista.

En cada casa, los investigadores encuestaron sobre la tenencia de la tierra (dentro y fuera del PTT), actividades agrícolas, agua, vivienda, tamaño y composición de la familia, educación materna y fechas de nacimiento de los niños menores de 5 años. Donde dos o más familias compartían la vivienda, se suponía que también compartían el trabajo agrícola.

Se registró el peso de todos los niños menores

11. V. Aguayo, "Nutrición en El Salvador: la prioridad del nuevo milenio", presentación realizada con motivo de la Conferencia Nacional de Nutrición de El Salvador, San Salvador, 14-15 enero de 1999.

12. R. Martorell, L. Kettel Khan, D. Schroeder, "Reversibility of stunting: epidemiological findings in children from developing countries", Eur J Clin Nutr, 48 (suppl 1), 1994, pp. S45-S57.

13. I. Rawson, V. Valverde, "The etiology of malnutrition among preschool children in rural Costa Rica", $J$ Trop Pediatr, 22, 1976, pp. 12-17.

14. G. Gutiérrez, R. Tapia Conyer, H. Guiscafré et al, "Impact of oral rehydration and selected public health interventions on reduction of mortality from childhood diarrhoeal diseases in Mexico", Bull World Health Organ, 74, 1996, pp. 189-197.

15. E. Hertz, J. Hebert, J. Landon, "Social and environmental factors and life expectancy, infant mortality, and maternal mortality rates: results of a cross-national comparison", Soc Sci Med, 39, 1994, pp. 105-114.

16. V. Valverde, R. Martorell, V. Mejía-Pivaral et al, "Relationship between family land availability and nutritional status", Ecol Food Nutr, 6, 1977, pp. 1-7.

17. J. Briscoe, "Water supply and health in developing countries: selective primary health care revisited", $A m \boldsymbol{J}$ Public Health, 74, 1984, pp. 1009-1013.

18. M. Yusuf, A. Ehrlich, G. Maritim, "A comparison of nutritional status of pre-school children of cooperative and individual farmers in rural Ethiopia", Trop Geogr Med, 45, 1993, pp. 93-94.

19. D. Pelletier, L. Msukwa, "The use of national sample surveys for nutritional surveillance: lessons from Malawi's national sample survey of agriculture", Soc Sci Med, 32, 1991, pp. 887-898.

20. Una parte de los datos que se presentan aquí aparecieron por primera vez en JAMA, Journal of the American Medical Association (Chicago, Illinois, Estados Unidos), Vol. 281, 2, el 13 enero de 1999, pp. 184-90. 
de 5 años en múltiplos de $100 \mathrm{~g}$ (vestidos con ropa ligera) y la talla en milímetros (mayores de 2 años de pie, menores en decúbito supino), según protocolos internacionales ${ }^{21}$. Cuatro niños no permitieron que los pesáramos ni midiéramos.

Se calcularon los índices estandarizados (Z-scores $^{22}$ ) de talla-para-edad, peso-para-talla y pesopara-edad en cada niño, utilizando las curvas de referencia internacional de la Organización Mundial de la Salud y los Centros para el Control y la Prevención de la Enfermedad de Atlanta, Estados Unidos (programa Epi-Info) ${ }^{23}$. Siguiendo criterios internacionales, se definió baja talla como un Zscore de talla-para-edad menor de -2 , bajo peso como un Z-score de peso-para-edad menor de -2 , y emaciación como un Z-score de peso-para-talla menores de -2 . Dos valores considerados poco probables (Z-score $\geq 6$ ó $\leq-6$ ) se descartaron y no figuraron en los análisis estadísticos. Se utilizó la prueba estadística de Chi-cuadrada para evaluar la función del azar en las asociaciones encontradas entre factores socioeconómicos y baja talla. Además, se entrevistaron a directivos y promotores de salud de las comunidades, con el fin de obtener información sobre despoblación, repoblación, agua, saneamiento, viviendas, escuelas, caminos, salud y tenencia de la tierra.

El protocolo del estudio fue aprobado por Médicos por los Derechos Humanos (Physicians for Human Rights, Boston, Massachusetts, Estados Unidos). Los padres de familia y directivos entrevistados dieron su consentimiento verbal. El equipo de investigadores se comprometió a no revelar los nombres de las familias ni los de las comunidades sin la autorización de los directivos o padres de familia entrevistados.

En enero de 1999, después de analizar los datos recabados en la investigación, el equipo de investigadores presentó los hallazgos a un grupo conformado por médicos, enfermeras, promotores de salud, parteras y representantes de organismos no gubernamentales del municipio estudiado, que discutieron los resultados y sus implicaciones.

\section{Resultados y comentarios}

\subsection{Desnutrición infantil}

Visitamos 761 niños en 458 viviendas. La prevalencia global de baja talla-para-edad fue del 32.4 por ciento, la de bajo peso-para-talla del 1.3 por ciento, y la de bajo peso-para-edad del 9.2 por ciento. No encontramos diferencias estadísticamente significativas entre sexos (la prevalencia de baja talla en los niños fue de 33.9 por ciento y en las niñas, de 30.8 por ciento). La prevalencia de baja talla aumentaba con la edad: 7.0 por ciento en menores de 6 meses, incrementándose hasta 41.6 por ciento en el quinto año de vida. El aumento de la prevalencia de baja talla con la edad es un patrón esperado: cuanto más tiempo se encuentra el niño sin alimentos $y / 0$ con infecciones, se acumula un mayor retardo en el crecimiento.

La figura 2 muestra la distribución de tallapara-edad en el municipio estudiado, comparada con la distribución de referencia internacional. Como se puede observar, la distribución de tallapara-edad en el municipio tiene la misma forma que la distribución de referencia, pero está desplazada a la izquierda con respecto a ésta. Esto significa que no sólo la media de talla es más baja en esta población, sino que los niños más altos de esta población son más bajos que los niños más altos de la población de referencia (la población "normal").

Figura 2

\section{Distribución de Z-Score para talla baja}

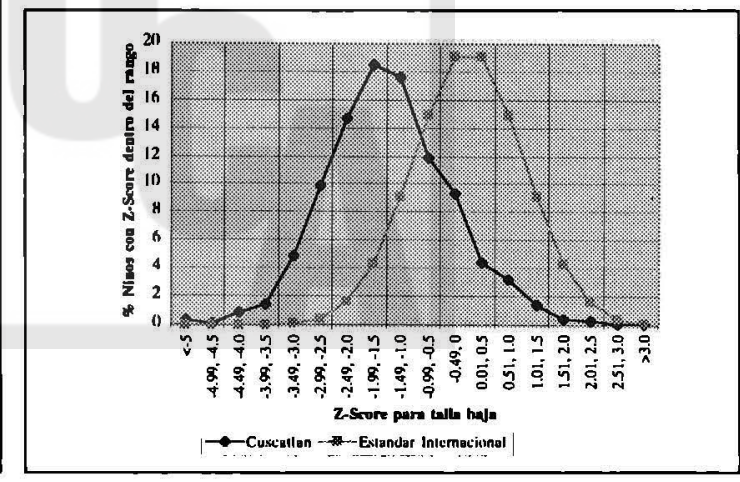

21. World Health Organization Expert Committee on Physical Status, op. cit.

22. En una población "normal", la media del Z-score es cero, y el $95 \%$ de los niños tienen Z-scores entre -2 y + 2 .

23. Epi Info Version 6 [computer program] Atlanta, Georgia, Estados Unidos: Centers for Disease Control and Prevention, 1994. 
La prevalencia general de baja talla en las comunidades que estudiamos es significativamente más alta que la prevalencia nacional de baja talla en El Salvador, según los datos preliminares de FESAL-98, pero es consistente con la prevalencia de baja talla encontrada por FESAL-98 en Cuscatlán. Según datos preliminares, la prevalencia de baja talla en Cuscatlán ( 35 por ciento) en 1998 era más alta que la prevalencia en cualquier otro departamento de El Salvador, y más del doble de la prevalencia en el departamento de San Salvador, donde era del 16 por ciento.

Las comunidades investigadas eran muy heterogéneas y la prevalencia de baja talla varió mucho entre ellas. En la comunidad con mayor desnutrición infantil crónica la prevalencia fue del 59 por ciento, casi el doble de la prevalencia general en nuestro estudio, y casi el triple de la preva- lencia nacional según FESAL-98. Para ver estas cifras en un contexto más amplio, cabe mencionar que la prevalencia de baja talla en Corea del Norte (considerado uno de los 10 países con más desnutrición crónica a nivel mundial) fue del 62 por ciento en $1998^{24}$. No obstante, la variabilidad entre comunidades con respecto a la prevalencia de baja talla debe interpretarse con cuidado, ya que el relativamente bajo número de niños medidos en cada comunidad hace difícil excluir que las diferencias se deban al azar.

\subsection{Desnutrición, tenencia de la tierra y agri- cultura}

Aunque la población que visitamos era pobre, encontramos mucha heterogeneidad en los patrones de tenencia de la tierra y en las actividades agrícolas (ver Cuadro 1).

\section{Cuadro 1}

Características de las viviendas

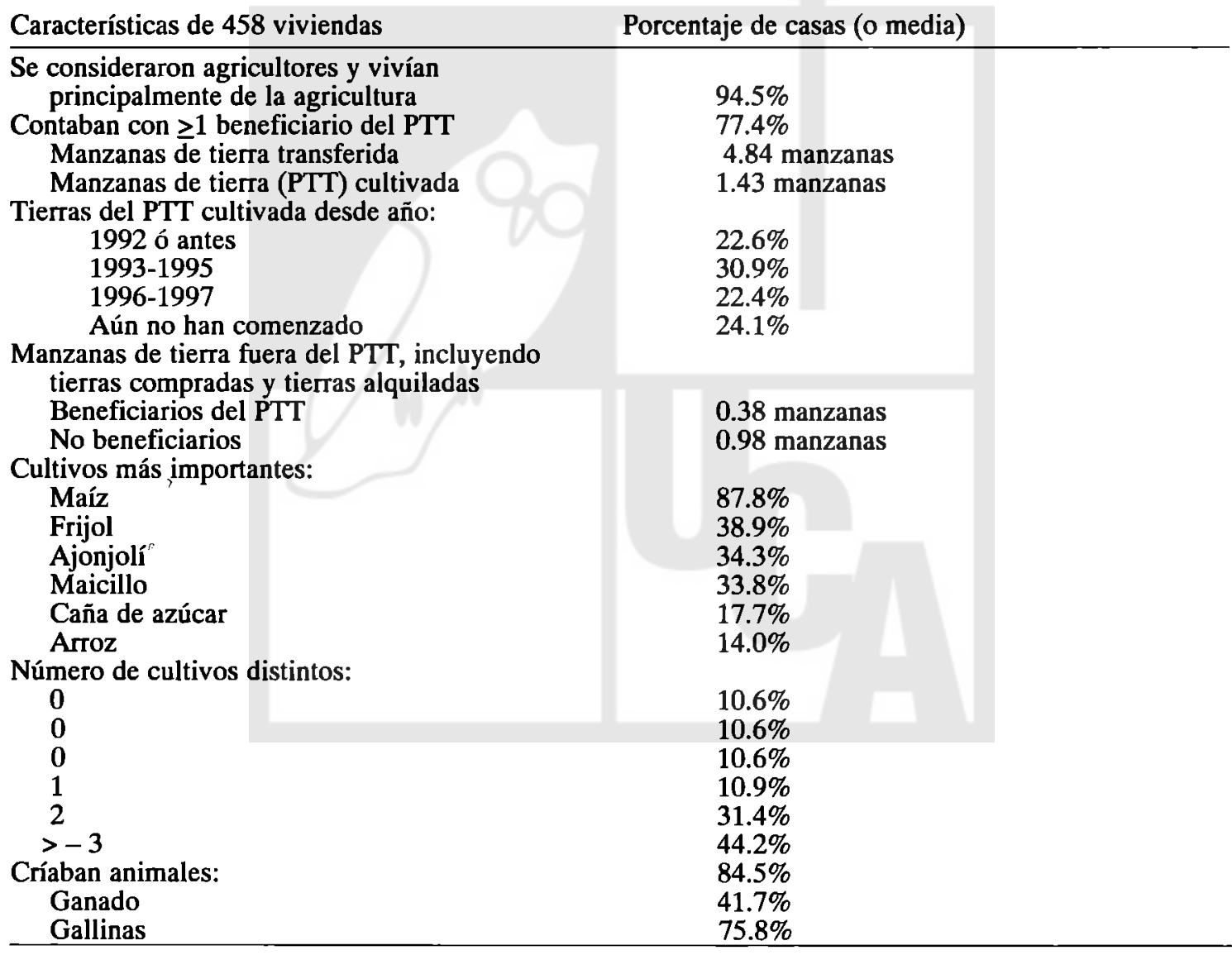

24. E. Rosenthal, "In North Korean hunger, legacy is stunted children", New York Times, 10 de diciembre 1998, p. 1. 
La mayoría de las casas que visitamos contaban con por lo menos un beneficiario del Programa de Transferencia de Tierras (PTT). Cada beneficiario había recibido, o iba a recibir, un promedio de casi 5 manzanas de tierra. De éstas, se estaba cultivando menos de la tercera parte en promedio. La mayoría (61.3 por ciento) de las familias que recibieron tierras del PTT estaban cultivando 1.0 manzana o menos de las tierras transferidas. Sólo 1.9 por ciento de las familias inscritas en el Programa cultivaban 5 manzanas o más de las tierras transferidas (ver Figura 3). Según esto, la mayoría de las familias que eran beneficiarios del Programa de Transferencia de Tierras aún estaban trabajando como agricultores de subsistencia —casi igual que las familias que no recibieron tierras en la transferencia - y la cantidad de tierras legalmente transferidas no parece un indicador confiable de la situación actual del agricultor.

Figura 3

\section{Manzanas de tierra trabajadas del PTT}

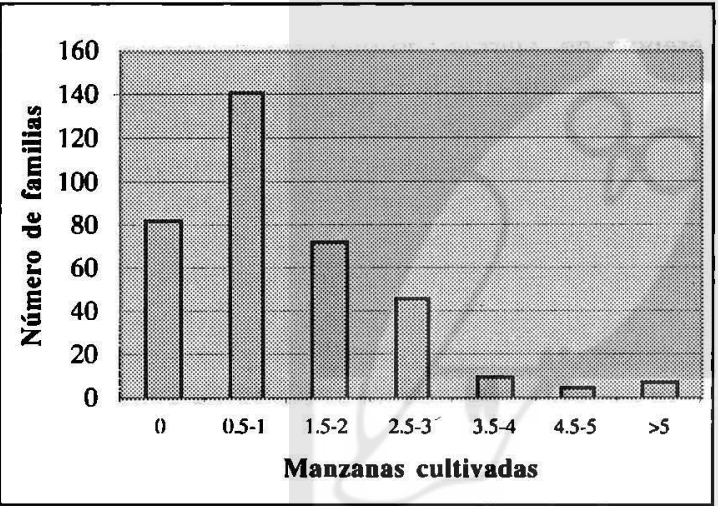

Preguntamos a los padres de familia por qué no cultivaban todas las tierras transferidas y las respuestas incluyeron: $(a)$ falta de recursos económicos para la compra de insumos; $(b)$ alto costo de créditos; (c) tierra inservible para cultivar; $(d)$ tierra localizada tan lejos de la comunidad que no se podía cultivar sin abandonar esta última; $(e)$ falta de repartición de parcelas particulares; $(f)$ falta de hombres en la casa; $(g)$ falta de tractores, bueyes, etc. y $(h)$ aún no se había localizado tierra para comprar.

Un año después, trabajadores sanitarios y representantes de organismos no gubernamentales locales agregaron otras posibles explicaciones: (a) las técnicas antiguas, adecuadas para el cultivo de

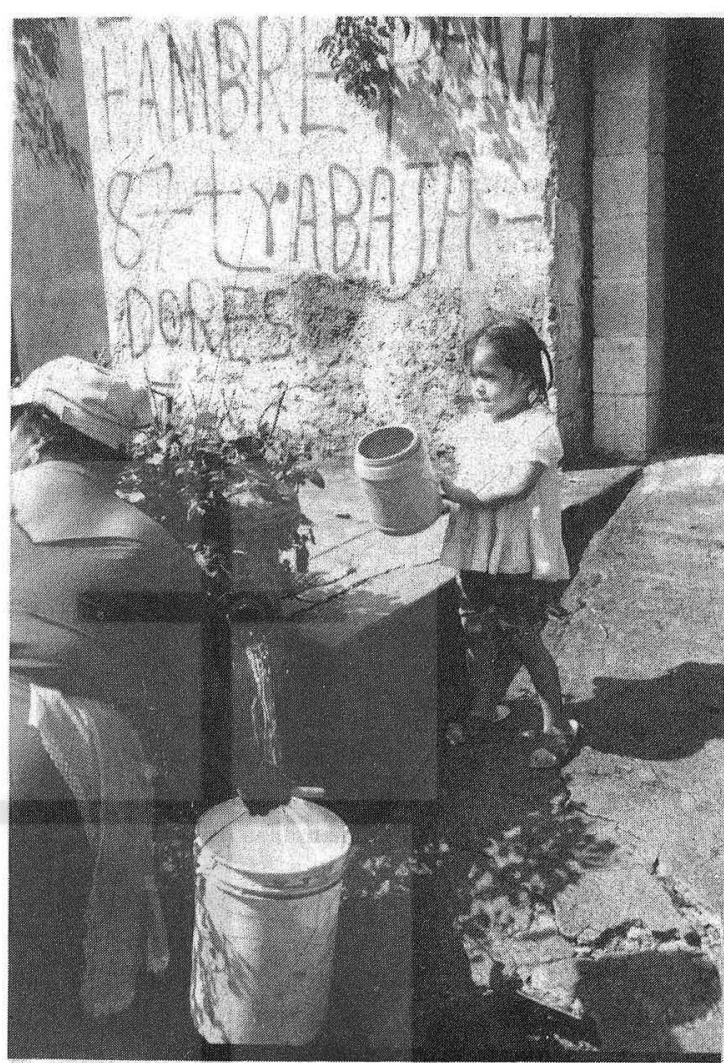

maíz y frijol, ya no funcionan para los nuevos cultivos que se requieren en la actualidad. (b) La relación entre precios de insumos/créditos y beneficios por la venta de productos agrícolas hace más rentable el abstenerse de cultivar. (c) Falta de riego. (d) El alto nivel de delincuencia invita a no parecer demasiado próspero, por temor a ser robado. (e) Muchas personas han tenido que vender sus tierras para pagar los gastos de cirugía de sus enfermos (u otra emergencia familiar). $(f)$ Muchas personas no han sabido aprovechar la asesoría técnica que han recibido. (g) Alto riesgo de pérdidas si la inversión en la compra de insumos no se acompaña de condiciones meteorológicas favorables. En muchos casos (más de 20 por ciento de las familias inscritas en el Programa de Transferencia de Tierras) no estaban cultivando nada de las tierras transferidas y vivían como jornaleros o arrendaban otras tierras.

Los análisis estadísticos no revelaron ninguna relación significativa entre el número de manzanas de tierra poseídas por la familia y la desnutrición crónica infantil (baja talla) de sus niños, excepto en los niveles de tenencia de tierra más altos. Sin 
embargo, sí encontramos una fuerte relación entre el número de manzanas activamente cultivadas y la desnutrición crónica infantil. Cuanto mayor era el número de manzanas cultivadas por la familia, menor era la prevalencia de desnutrición en sus niños (ver Figura 4).

\section{Figura 4}

Talla baja y tierras del PTT

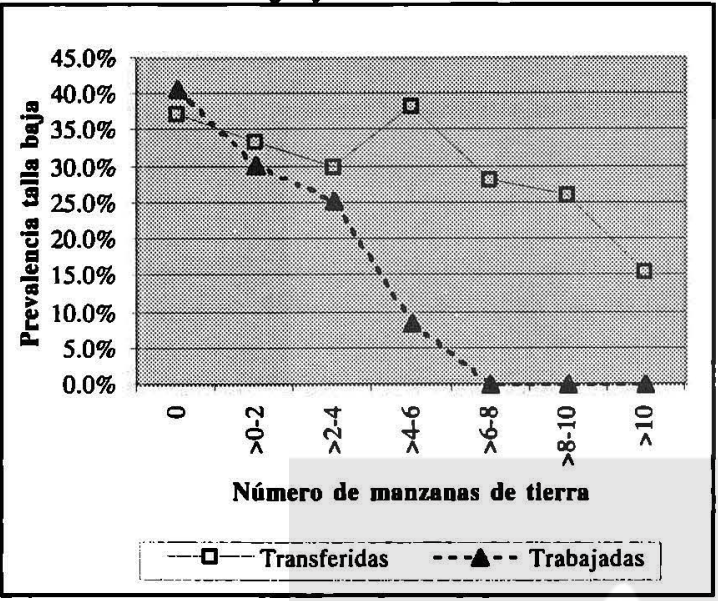

La diversificación del trabajo agrícola también estaba asociada a un mejor estado de salud de los niños. Cuantos más cultivos distintos producía la familia, menos desnutrición infantil encontramos (ver Figura 5). Las familias que no cultivaban tierras propias (por ejemplo, donde solamente trabajaban como jornaleros en tierras ajenas) tenían los niños más desnutridos; mientras que las familias que trabajaban 3 ó más cultivos distintos, tenían los niños menos desnutridos. La mayoría de las familias que practicaban el monocultivo -56 por ciento- sólo sembraban maíz.

\section{Figura 5}

Variedad de cultivos y talla baja

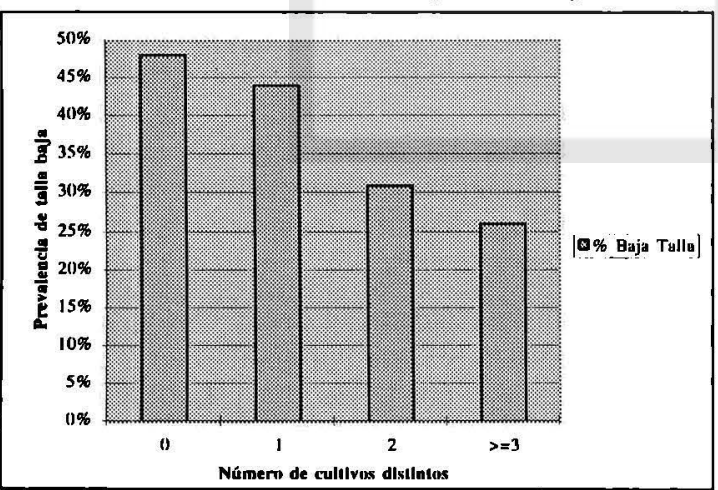

Además, las familias que agregaban la cría de animales (pequeños, como gallinas, o grandes, como ganado) a sus actividades tenían niños con menor grado de desnutrición (ver Figura 6).

Figura 6

Cría de animales y talla baja

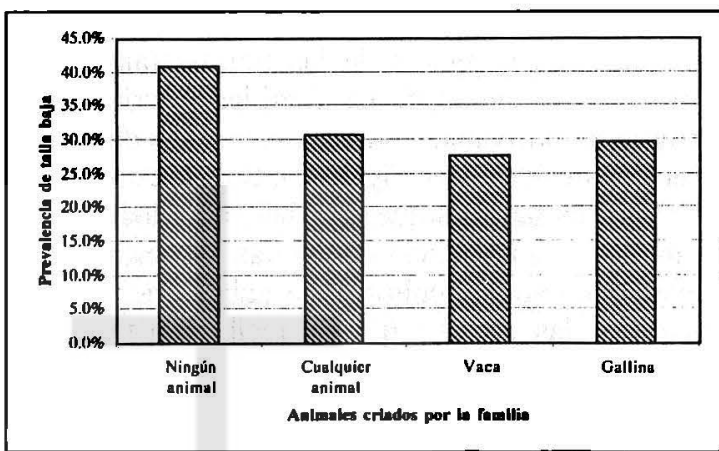

Las dos relaciones descritas -manzanas trabajadas/baja talla, número de cultivos/baja talla— también tenían un componente temporal. Las familias que accedieron antes a las tierras del Programa de Transferencia de Tierras (porque comenzaron a trabajar sus tierras antes de la compra legal, o porque recibieron sus títulos en los primeros años del programa) también fueron las que tenían mayor diversidad de cultivos y trabajaron más manzanas (ver Figura 7).

\section{Figura 7}

Tierras cultivadas del PTT y variedad de productos cultivados, por año de comienzo del cultivo

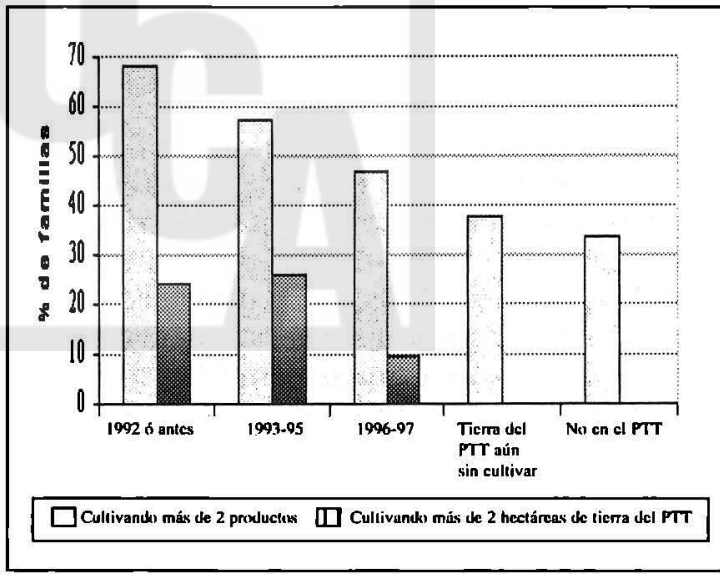

No podemos establecer con certeza por qué observamos estas relaciones entre tiempo de acceso a 
las tierras, cantidad de tierra cultivada, diversificación de cultivos y desnutrición. Algunas posibles explicaciones son: (1) Las familias que previamente recibieron sus tierras tuvieron la ventaja de comenzar el trabajo agrícola durante la época en que hubo más ayuda internacional (asesoría de agrónomos, créditos, insumos, etc.) y contaron con más recursos al principio. (2) Cada año de trabajo en la parcela proporciona al agricultor más tiempo para limpiar, cercar, abonar y mejorar las tierras en general. (3) Las primeras tierras compradas fueron las mejores. (4) Los precios de los insumos eran tan altos en comparación con el precio del maíz en el mercado, que las familias que sólo cultivaban este último no ganaban dinero suficiente para alimentar bien a los niños o mejorar sus tieras. (5) Cuantos más cultivos y animales, más variedad en la dieta de los niños y más posibilidades de ganar dinero a través de la venta de productos. (6) Las familias que cultivaban más tierras eran también las más activas o las mejores desde el punto de vista económico o político y, por tanto, aquellas cuyos niños tenían un menor riesgo de desnutrición.

\subsection{Desnutrición y agua}

Después de la relación entre tierras trabajadas y desnutrición, la relación entre acceso al agua y desnutrición fue la más importante que observamos. En realidad, ninguna familia tenía acceso a agua potable. Sólo el $\mathbf{6 5 . 0}$ por ciento de las familias tenían acceso al agua de tubería (conexión domiciliar o comunal). De los sistemas de agua que aún funcionaban, muchos eran sistemas temporales instalados por ACNUR, la CICR u organismos no gubernamentales durante la guerra. El racionamiento del agua fue casi la norma, sobre todo en el verano.

Figura 8

Acceso al agua de tubería y talla baja

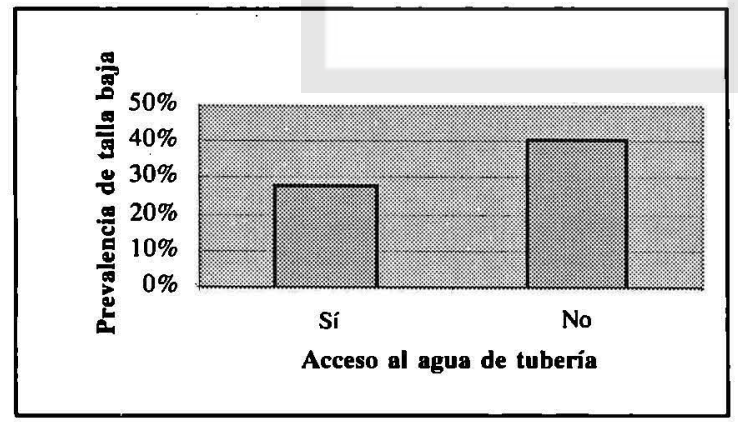

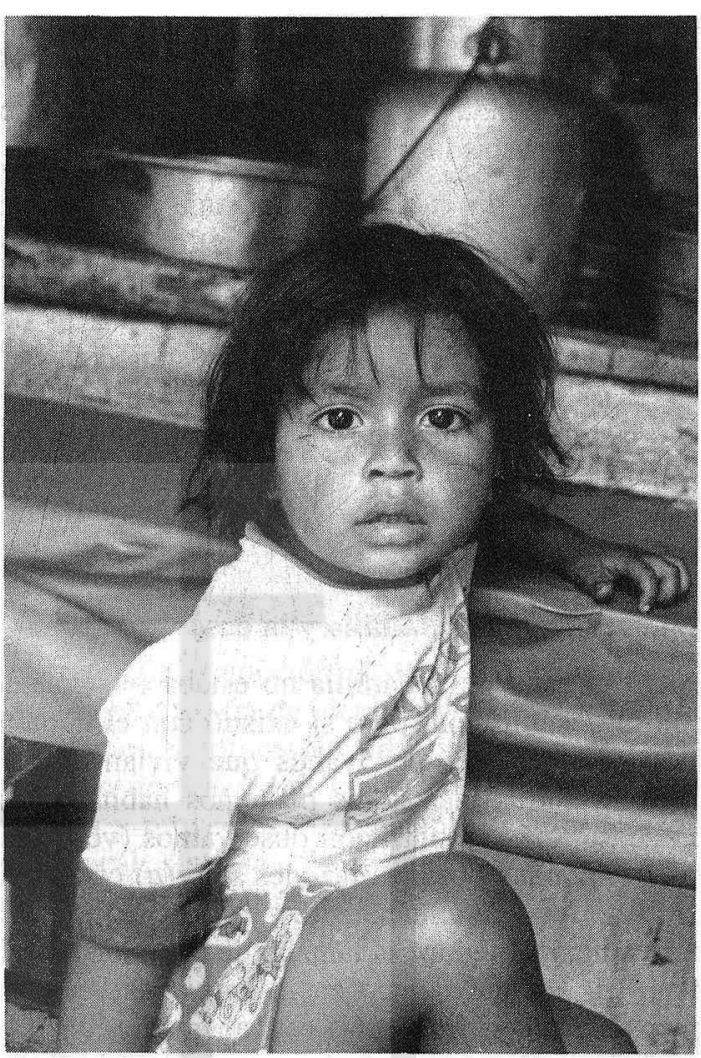

Donde no había sistema de agua de tubería encontramos más desnutrición (ver Figura 8). Además, las comunidades que recibieron sus sistemas de agua más temprano tenían menos niños con baja talla, a pesar de la ausencia de agua potable y el racionamiento del agua que afectaba a tantas comunidades. Casi la mitad de los niños que se han estudiado vivían en comunidades sin sistema de agua. No podemos decir, en definitiva, por qué existe esta relación tan estrecha entre la presencia de sistemas de agua y la ausencia de desnutrición, pero algunos factores probables son: (a) la madre que no tiene necesidad de ir a buscar agua tiene más tiempo para cuidar a sus nin̄os y/o producir alimentos. (b) Donde existe acceso al agua es más fácil mantener la higiene y, de esta manera, prevenir la diarrea y otras enfermedades que debilitan al niño pequeño. (c) Cuando el acceso al agua es cercano, es factible regar huertas y árboles frutales, aumentando así la calidad de la dieta y los ingresos familiares. (d) Los niños que no tienen necesidad de buscar y transportar el agua, no gastan sus calorías en estas actividades.

La falta de sistemas de agua contribuyó al fracaso de otros programas de ayuda. Por ejemplo, la 
falta de agua para el riego de árboles frutales y para el consumo de vacas lecheras, liquidó proyectos cuya meta era mejorar la alimentación y los ingresos de estas familias.

En el estudio no se encontró una relación significativa entre la presencia de letrina y el estado nutricional, aunque advertimos una leve disminución de la desnutrición donde había letrina. Es posible que la presencia de ésta (75.9 por ciento de las casas tenían letrinas) no garantizara su uso, o que la relación entre letrina y desnutrición, relativamente débil, estuviera ensombrecida por la relación, más estrecha, de desnutrición con tierras, cultivos y agua.

\subsection{Desnutrición, familia y la casa}

El tamaño de la familia no estaba relacionado con la desnutrición, pero sí existió con el número de niños menores de 5 años que vivían en una casa. Mientras más niños pequeños habitaban en una casa, más desnutrición observamos (ver Figura 9). Algunas posibles razones son: (a) cuando la cantidad total de alimentos disponibles es fija, cada niño adicional reduce el tamaño de la ración individual. (b) La mujer que tiene hijos varias veces en pocos años, con frecuencia está anémica y débil, y sus niños, además, pueden nacer desnutridos o no beneficiarse de una lactancia adecuada. (c) Cuando un elevado número de niños pequeños viven en una sola casa, esto puede significar que dos o más madres jóvenes vivan bajo el mismo techo, muchas veces a causa de la falta de recursos para adquirir una casa propia o para el grupo familiar.

Figura 9

Niños menores de 5 años en la casa y la talla baja

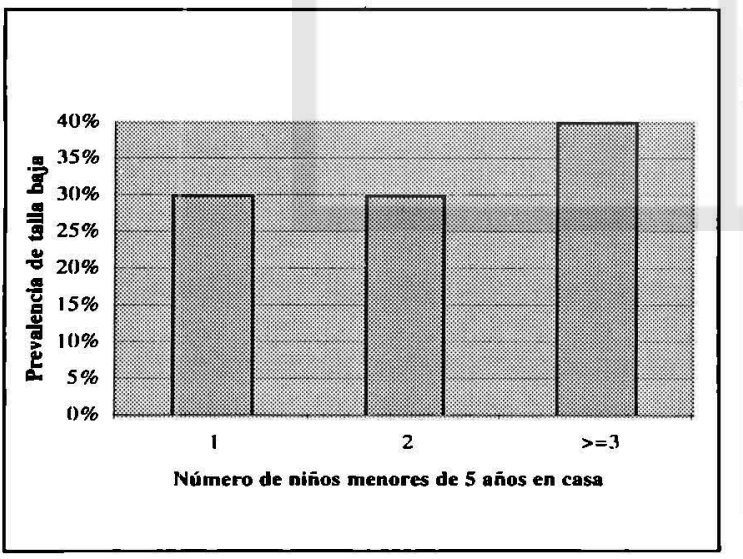

Tanto los niños de madres con mayor nivel educativo, como aquellos que vivían en casas más sólidas (por ejemplo, de bloque o ladrillo) tenÌan menor grado de desnutrición, aunque estas relaciones no eran muy estrechas.

\section{Cuadro 2}

Características de familias y casas

Características

Porcentaje de casas

(o media)

Edad de la madre:

27.6 años

Educación materna:

Sin estudios

$25.3 \%$

$1^{\circ}$ a $4^{\circ}$ grado

$52.4 \%$

$5^{\circ}$ grado o más

$22.3 \%$

Padre de los niños que vive

con la madre:

$78.1 \%$

Número de personas que

viven en la casa:

6.3 personas

Número de niños menores de

5 años que viven en la casa:

Tipo de construcción de la casa

Plástico o lámina

1.8 nin̄os

Paja, bahareque

$12.4 \%$

$8.2 \%$

Adobe

$35.8 \%$

Ladrillo o bloque de cemento

$43.6 \%$

\subsection{Desnutrición y Programas de Salud}

En el estudio no encontramos relación entre la presencia de un promotor de salud en la comunidad y la prevalencia de desnutrición crónica infantil. Tampoco detectamos relación entre la presencia de ciertos programas de los promotores de salud y la desnutrición. Otros estudios afirman haber encontrado efectos positivos (datos no publicados). Es difícil decir con certeza por qué el efecto sobre la desnutrición no fue evidente. Algunas explicaciones posibles incluyen:

(1) No pudimos cuantificar el trabajo realizado por los promotores de salud que ya no están activos en las comunidades. Casi todas las comunidades contaban con equipos de promotores en la época de repoblación; los promotores que ya no laboraban, pudieron haber dejado efectos de su trabajo a largo plazo.

(2) Muchos promotores de salud atienden a personas que no viven en sus comunidades. Asi- 
mismo, las comunidades que no tenían sus propios promotores pudieron ser atendidos por los de sus comunidades vecinas. Por ejemplo, las campañas de vacunación y el programa de parteras de la región brindaron atención a muchas comunidades que no contaban con promotores activos.

(3) No pudimos controlar la calidad de los promotores ni el contenido específico de su trabajo. No existe una definición estándar de "promotor de salud".

(4) Últimamente, los promotores no han contado ni con los materiales ni con el respaldo profesional que anteriormente tenían. Es posible que su trabajo sea ahora menos eficaz.

(5) El posible efecto de "promoción" puede ser muy limitado donde hay escasez absoluta de alimentos y agua.

En definitiva, el diseño de nuestro estudio no permite extraer conclusiones firmes sobre el impacto de los programas de los promotores de salud. No obstante, este tema merecería su propio estudio.

\section{Recomendaciones}

Cuando presentamos los hallazgos de nuestro estudio a los trabajadores de la salud y representantes de los organismos no gubernamentales de la región estudiada, les preguntamos: “¿por qué hay tanta desnutrición crónica aquí?". Sus respuestas fueron de dos tipos: la primera, a causa de la escasez absoluta de recursos (por ejemplo, tierras cultivables); la segunda, por la mala gestión de los recursos disponibles (por ejemplo, la familia que compra charamuscas o aguardiente en lugar de comprar aceite o abono; o el agricultor que sólo cultiva productos sin valor en el mercado).

Posteriormente les solicitamos sus recomendaciones para futuras acciones. Estas fueron algunas de sus sugerencias: (1) incorporación del sector agrícola a los planes de desarrollo nacional y municipal; (2) mejora de la organización comunal; (3) reducción de la tasa de interés para créditos agrícolas; (4) disponibilidad de un seguro para cosechas perdidas (como en el caso del huracán Mitch); (5) promoción exclusiva de la lactancia

materna; (6) promoción de la planificación familiar; (7) promoción de técnicas de conservación de alimentos; (8) promoción de proyectos de agua, para consumo humano y riego; (9) promoción de cultivos y proyectos más rentables que el maíz, a nivel local, sin forzar la emigración hacia la zona metropolitana; (10) promoción de un mejor manejo de los recursos familiares.

A las recomendaciones de la gente afectada por los problemas descritos en nuestra investigación, solamente quisiéramos agregar lo siguiente: (1) promoción del uso del indicador talla-paraedad en la vigilancia de niños y en el estudio del impacto de los programas de desarrollo. (2) Realizar estudios similares a éste en otras regiones de El Salvador, con el fin de evaluar si los mismos patrones existen en otros lugares. La existencia de variaciones locales puede requerir de la adopción de soluciones flexibles. (3) Efectuar otros estudios para aclarar otras cuestiones importantes, como ¿cuál sería el proceder más eficaz de los promotores de salud? ¿Cuál debe ser el orden de implementación de los proyectos? (¿primero se hace la huerta que necesita riego, o el sistema de agua que la riega?). ¿Cuál sería la forma más eficaz de ayudar al pequeño agricultor para que pueda aprovechar todas su tierras?

\section{Conclusión}

A principios de 1998, seis años despues de firmar los acuerdos de paz de Chapultepec, una población de beneficiarios del proceso de reconstrucción de la posguerra vivía aún en la pobreza absoluta. Aunque la mayoría recibió tierras a través del Programa de Transferencia de Tierras (PTT), esto 
no fue suficiente para garantizar una supervivencia digna de sus familias. La mayoría aún trabajaban una manzana o menos (una agricultura que ni alcanza para la subsistencia ${ }^{25}$ ) y nadie tenía agua potable. La prevalencia de desnutrición crónica infantil era aún muy alta y existía una relación entre demoras en el proceso de reconstrucción (fecha de primer cultivo de tierras repartidas, fecha de instalación de sistema de agua) y la desnutrición. El diseño de nuestro estudio no nos permite establecer si las demoras causaron la desnutrición, o si las demoras y la desnutrición tenían causas comunes no identificadas. Sin embargo, sospechamos que la relación es de causa-efecto. En cualquier caso, la alta prevalencia de desnutrición crónica infantil tiene implicaciones alarmantes para el futuro bienestar y productividad de la población afectada.

El Programa de Transferencia de Tierras no fue diseñado como un programa de salud, pero sus efectos en este campo, al igual que los efectos del abastecimiento de agua, son muy importantes. Quien vigile la situación de salud del niño salva- doreño tendrá que tomar en cuenta todo factor que afecte a la salud, sea o no identificado como intervención médica.

Aunque la guerra y la primera etapa de reconstrucción de la posguerra han terminado, esperamos que los hallazgos de estudios como éste puedan utilizarse para diseñar un futuro programa de desarrollo, que responda específicamente a la realidad actual salvadoreña, con su propia mezcla de problemas de tierra, agua, ecología y salud.

\section{Agradecimientos}

Nuestro agradecimiento a todos los niños, padres de familia, parteras y promotores de salud que colaboraron en este estudio. A los Médicos por los Derechos Humanos, Socorro Luterano Mundial, y el Fondo para el Socorro Médico Internacional por su apoyo financiero. A quienes nos ayudaron con sus conocimientos respecto a agua, tierras y salud.

2 marzo 1999.

25. Según la FAO, algunas fuentes han calculado que una familia campesina necesita 7.35 manzanas para salir de la pobreza absoluta, y 19.1 manzanas para salir de la pobreza relativa. 